\title{
Preface to the Special Issue Dedicated to Marco A. López's 70th Birthday
}

\section{Convex and Nonconvex Analysis, and Semi-Infinite Optimization: A Special Tribute to the Work of Marco López Cerdá}

\author{
Maria Josefa Cánovas ${ }^{1}$ - Alexander Kruger ${ }^{2}$ • Hoang Xuan Phu ${ }^{3}$ - Michel Théra ${ }^{2,4}$
}

Published online: 27 May 2020

(C) Vietnam Academy of Science and Technology (VAST) and Springer Nature Singapore Pte Ltd. 2020

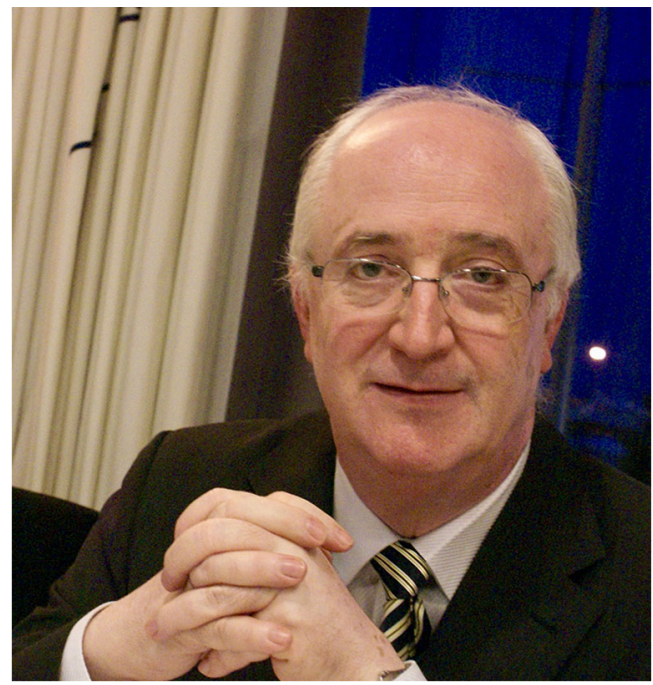

Professor Dr. Marco Antonio López Cerdá

This two-part issue of Vietnam Journal of Mathematics is dedicated to the life and work of Marco López Cerdá and is devoted to several topics of set-valued and variational analysis in which Marco has had interest and mainly worked for several decades.

The contents of this special issue that Vietnam Journal of Mathematics publishes as a tribute to Marco López Cerdá is described below. It contains 20 research papers contributed by highly distinguished mathematicians, colleagues and friends of Marco López Cerdá. These contributions cover topics in both theoretical and applied aspects and show the state

Michel Théra

michel.thera@unilim.fr 
of the art in many directions of the areas in which Marco has made major contributions. Some of the authors were Marco's students or have collaborated with him at some time of his career and all of them want to show their appreciation and respect to Marco and his work developed along the years. A detailed presentation of the life and mathematical contributions of Marco is given by the editors in the first article of this special issue and there is no need to recall here the biography, academic career and Marco's outstanding contributions.

All submissions have passed through the usual rigorous reviewing process. We would like to thank warmly the authors and reviewers as well as Ms. Nguyen Thi Hue from the Editorial office of Vietnam Journal of Mathematics for their work.

The first contribution of this Special Issue is a presentation of Marco's life and mathematical results by Maria Josefa Cánovas, Alexander Kruger, Hoang Xuan Phu and Michel Théra.

The paper "On the Existence of Solutions and Tikhonov Regularization of Hemivariational Inequality Problems" by Guo-ji Tang, Zhongping Wan and Xianfu Wang is devoted to the existence of solutions and Tikhonov regularization theory for a class of hemivariational inequalities, when the mappings satisfy the so-called hemivariational inequality property and a rather weak coercivity condition.

The main focus of "McShane's Extension Theorem Revisited" by Gerald Beer concerns a new proof of the McShane extension theorem for bounded uniformly continuous real-valued functions on a metric space based on a well-known extension theorem for real-valued Lipschitz functions and uses McShane's theorem to give an elementary proof of the equivalence of the most important characterizations of metric spaces in which the real-valued uniformly continuous functions form a ring.

"Convergence Rate of Inertial Proximal Algorithms with General Extrapolation and Proximal Coefficients" by Hedy Attouch, Zaki Chbani and Hassan Riahi analyzes the convergence rate of the inertial proximal algorithms, which can be interpreted as the discrete time version of inertial continuous gradient systems with general damping and time scale coefficients. They show the fast convergence of values and the convergence of iterates.

The contribution "Geometric and Metric Characterizations of Transversality Properties" by Hoa T. Bui, Nguyen Duy Cuong and Alexander Y. Kruger continues their study of 'good arrangements' of collections of sets near a point in their intersection and clarifies quantitative relations between several geometric and metric characterizations of the transversality properties of collections of sets and the corresponding regularity properties of set-valued mappings.

Christoph Neumann, Oliver Stein and Nathan Sudermann-Merx study in their contribution "Bounds on the Objective Value of Feasible Roundings" how for mixed-integer linear and nonlinear optimization problems the optimal value deviates from the objective value of feasible points which are constructed by the feasible rounding approach suggested previously by the authors. Computational results for large scale knapsack problems illustrate their theoretical results, in particular with respect to the effect of integer grid fineness.

The paper "Subdifferentials and Stability Analysis of Feasible Set and Pareto Front Mappings in Linear Multiobjective Optimization" by Maria Josefa Cánovas, Marco A. López, Boris S. Mordukhovich and Juan Parra concerns multiobjective linear optimization problems in $\mathbb{R}^{n}$, which are parameterized with respect to the right-hand side perturbations of inequality constraints, and focuses on measuring the variation of the feasible set and the Pareto front mappings around a nominal element while paying attention to some specific directions. 
The contribution "An Asymptotic Mean Value Formula for Eigenvalues of the Hessian Related to Concave/Convex Envelopes" by Pablo Blanc and Julio D. Rossi establishes an asymptotic mean value property that characterizes viscosity solution to certain fully nonlinear PDEs associated with the eigenvalues of the Hessian.

In "Lagrange Multipliers in Convex Set Optimization with the Set and Vector Criteria", Lidia Huerga, Bienvenido Jiménez and Vicente Novo study, in the setting of normed spaces ordered by a convex cone, a convex set-valued optimization problem where the feasible set is given by a cone-constraint. They derive Lagrange optimality conditions for weakly efficient solutions of the problem following both the set and the vector solution criterion, by using a scalarization technique based on a nonlinear scalarizing functional defined in terms of the oriented distance.

In the paper "Using Positive Spanning Sets to Achieve d-Stationarity with the Boosted DC Algorithm", Francisco J. Aragón Artacho, Rubén Campoy and Phan Tu Vuong combine the Boosted Difference of Convex functions Algorithm (BDCA) with a derivative-free optimization algorithm based on the use of positive spanning sets with the purpose of forcing the $d$-stationarity (lack of descent direction) at the point obtained. The BDCA is a recently proposed accelerated version of the classical DC Algorithm (DCA). The potential of this new approach is illustrated through some computational experiments on a Minimum-Sumof-Squares clustering problem, whose numerical results demonstrate that the new method provides better solutions while still remains faster than the DCA in the majority of test cases.

The focus of " $E$ '-Convex Sets and Functions: Properties and Characterizations" by Maria Dolores Fajardo and Jose Vidal concerns the analysis of e' -convex sets and functions that are the basis of the duality theory of evenly convex problems. The paper develops some of their most relevant properties and among them, characterizations and analytical properties that deeply relate these sets and functions with the notion of even convexity are given.

In "Weak Sequential Convergence in Bounded Finitely Additive Measures", Salvador López-Alfonso and Manuel López-Pellicer prove that boundedness and weak sequential convergence in the space of scalar bounded finitely additive measures on a $\sigma$-algebra $\Sigma$ follow from point boundedness and point weak convergence on small subsets of $\Sigma$. Several applications are provided.

"Approximate Proper Solutions in Vector Equilibrium Problems. Limit Behavior and Linear Scalarization Results" by César Gutiérrez concerns properties of approximate Benson and Henig proper efficient solutions of vector equilibrium problems. They provide relationships and the limit behavior for both kinds of solutions and the approximate weak efficient solutions of the problem. In addition, necessary and sufficient optimality conditions are derived via linear scalarization under generalized convexity assumptions.

"Selected Applications of Linear Semi-Infinite Systems Theory" by Miguel A. Goberna, Andrea B. Ridolfi and Virginia N. Vera de Serio shows how distinct branches of mathematics, Voronoi cells in computational geometry and approximate subdifferentials in mathematical analysis, can be naturally linked together and the results therein can be improved accordingly using the tools from linear semi-infinite systems. The paper completes the existing theory of farthest Voronoi cells of infinite sets of sites by appealing to well-known results on linear semi-infinite systems.

"Circumcentered Methods Induced by Isometries" by Heinz H. Bauschke, Hui Ouyang and Xianfu Wang studies the properness of the circumcenter mapping and the circumcenter method induced by isometries. The authors present weak convergence results for circumcentered isometry methods, which include the Douglas-Rachford method (DRM) and circumcentered reflection methods as special instances. 
In their contribution "Some Remarks on Greenberg-Pierskalla Subdifferentiability of Quasiconvex Functions", Michel Volle and Juan Enrique Martínez-Legaz observe that a quasiconvex function which is evenly quasiconvex at a point is not necessarily GreenbergPierskalla (briefly, G-P) subdifferentiable at that point, and prove that a quasiconvex function which is upper semicontinuous on the segments of its effective domain is G-P subdifferentiable on the relative interior of this effective domain. They give an application to surrogate duality in quasiconvex programming.

"Generalized Solutions of Quasi-Variational-Like Problems" by Truong Q. Bao, Niklas Hebestreit and Christiane Tammer deals with abstract quasi-variational-like problems, which cover vector quasi-variational problems with set-valued mappings, for instance. The aim of the paper is to show existence results for generalized solutions of quasi-variationallike problems. It focuses on the relationship between solutions of a quasi-variational-like problem and generalized solutions of an optimization problem minimizing the difference between inputs and outputs of the so-called variational selection. This approach allows to consider problems where the variational selection is non-convex and/or the underlying constraining set is unbounded.

In "An Elementary Proof of the Pontryagin Maximum Principle", Alexander D. Ioffe offers a proof of the maximum principle based on some ideas coming from variational analysis that allow to reduce the problem to one or a sequence of unconstrained minimization of Bolza-type functionals.

The focus of the contribution "Orbital Geometry and Group Majorisation in Optimisation" by Andrew Eberhard concerns the use of group symmetries in optimisation, in particular with respect to the structure of subdifferentials and projection operators. This allows him to study the normal cone structure of orbitopes associated with group majorisations, and using this framework to study projections and normal cones to sparsity constraints used in sparse signal recovery and compressed sensing.

In "Subdifferential of the Supremum via Compactification of the Index Set", Rafael Correa, Abderrahim Hantoute and Marco A. López give new characterizations for the subdifferential of the supremum of an arbitrary family of convex functions, dropping out the standard assumptions of compactness of the index set and upper semi-continuity of the functions with respect to the index. The proposed approach is based on the compactification of the index set, giving rise to an appropriate enlargement of the original family.

In the paper "Duality for Robust Linear Infinite Programming Problems Revisited", Nguyen Dinh, Dang Hai Long and Jen-Chih Yao establish robust strong duality results for many variants of a general robust linear infinite programming problem. Their approach follows from the analysis of a class of robust linear problem with convex conical constraints. Some results in the paper cover several known results in the literature, while others are new. Several robust Farkas-type results for linear infinite systems with uncertainty are obtained as by-products.

To conclude this preface, let us recall Marco's principal facets: he is a family man, plenty of love and affection for his wife Maria Pilar, their children and grandchildren; a wonderful friend; an outstanding mathematician; a great organizer.

March 31, 2020

Publisher's Note Springer Nature remains neutral with regard to jurisdictional claims in published maps and institutional affiliations. 


\section{Affiliations}

\section{Maria Josefa Cánovas ${ }^{1}$ • Alexander Kruger ${ }^{2}$ • Hoang Xuan Phu ${ }^{3} \cdot$ Michel Théra $^{2,4}$}

Maria Josefa Cánovas

canovas@umh.es

Alexander Kruger

a.kruger@federation.edu.au

Hoang Xuan Phu

hxphu@math.ac.vn

1 Center of Operations Research, Miguel Hernandez University of Elche, Elche, 03202 Alicante, Spain

2 Centre for Informatics and Applied Optimization, School of Science, Engineering and Information Technology, Federation University Australia, Ballarat, Vic, Australia

3 Institute of Mathematics, Vietnam Academy of Science and Technology, Hanoi, Vietnam

4 Laboratoire XLIM, UMR-CNRS n 7152, Université de Limoges, Limoges, France 